\title{
BMJ
}

\section{Comparison of rates of adverse events in adolescent and adult women undergoing medical abortion: population register based study}

\author{
Maarit Niinimäki, consultant gynaecologist, ${ }^{1}$ Satu Suhonen, chief physician, ${ }^{2}$ Maarit Mentula, consultant \\ gynaecologist, ${ }^{3}$ Elina Hemminki, research professor, ${ }^{4}$ Oskari Heikinheimo, chief physician, ${ }^{3}$ Mika Gissler, \\ research professor ${ }^{4,5}$
}

Department of Obstetrics and Gynecology, University Hospital of Oulu, Finland

${ }^{2}$ City of Helsinki Health Centre, Unit for Maternal and Child Health Care and Health Promotion, Helsinki, Finland

${ }^{3}$ Department of Obstetrics and Gynaecology/Kätilöopisto Hospital, Helsinki University Central Hospital, PO Box 610 00029 HUS, Helsinki, Finland

${ }^{4}$ National Institute for Health and Welfare, Helsinki, Finland

${ }^{5}$ Nordic School of Public Health, Gothenburg, Sweden

Correspondence to: 0 Heikinheimo oskari.heikinheimo@helsinki.fi

Cite this as: BMJ 2011;342:d2111 doi:10.1136/bmi.d2111

\section{ABSTRACT}

Objective To determine the risks of short term adverse events in adolescent and older women undergoing medical abortion.

Design Population based retrospective cohort study.

Setting Finnish abortion register 2000-6.

Participants All women $(n=27030)$ undergoing medical abortion during 2000-6, with only the first induced abortion analysed for each woman.

Main outcome measures Incidence of adverse events (haemorrhage, infection, incomplete abortion, surgical evacuation, psychiatric morbidity, injury, thromboembolic disease, and death) among adolescent ( $<18$ years) and older ( $\geq 18$ years) women through record linkage of Finnish registries and genital Chlamydia trachomatis infections detected concomitantly with abortion and linked with data from the abortion register for 2004-6.

Results During 2000-6, 3024 adolescents and 24006 adults underwent at least one medical abortion. The rate of chlamydia infections was higher in the adolescent cohort (5.7\% v 3.7\%, P 0.001). The incidence of adverse events among adolescents was similar or lower than that among the adults. The risks of haemorrhage (adjusted odds ratio $0.87,95 \%$ confidence interval 0.77 to 0.99 ), incomplete abortion $(0.69,0.59$ to 0.82$)$, and surgical evacuation $(0.78,0.67$ to 0.90$)$ were lower in the adolescent cohort. In subgroup analysis of primigravid women, the risks of incomplete abortion $(0.68,0.56$ to $0.81)$ and surgical evacuation $(0.75,0.64$ to 0.88$)$ were lower in the adolescent cohort. In logistic regression, duration of gestation was the most important risk factor for infection, incomplete abortion, and surgical evacuation.

Conclusions The incidence of adverse events after medical abortion was similar or lower among adolescents than among older women. Thus, medical abortion seems to be at least as safe in adolescents as it is in adults.

\section{INTRODUCTION}

Pregnancies among teenagers are mostly unplanned and offer a special challenge to family planning services. Most of all such pregnancies (up to $82 \%$ in the United States) are unintended. ${ }^{1}$ The decision to continue or terminate a pregnancy is strongly associated with age. Besides age, being a student or being single are important factors in young women's decisions on abortion. ${ }^{2}$ In the United States, $6 \%$ of all abortions are carried out in under $18 \mathrm{~s} .{ }^{1}$ In the United Kingdom, 9.5\% of abortions in 2009 were in adolescents. $^{3}$ Thus abortions among teenagers are common and are an important public health problem.

The medical termination of pregnancy using the antiprogestin mifepristone and a prostaglandin analogue has been widely established in several countries during the past decade. In 2009, $40 \%$ of abortions were medical in the United Kingdom. ${ }^{3}$ In Sweden and Finland the corresponding figures were $72 \%$ and $76 \%{ }^{45}$

Increasing use of medical termination of pregnancy points to a need for appropriate studies to confirm its safety in various target groups. Using nationwide register based data we showed that both medical and surgical abortions are generally safe, with few serious complications when gestation is less than 63 days. $^{6}$ The most common adverse events were haemorrhage and incomplete abortion. However, in that study we did not assess the safety of medical abortion among adolescents.

Data on the safety of medical abortion among adolescents are limited. In a small prospective study, medical abortion was found to be highly effective and well tolerated in adolescents aged 14 to 17 when gestation was less than 56 days. Initially, half of the participants experienced stress and fear, but these emotions improved significantly within the month after abortion. $^{?}$

In the present nationwide study we compared the safety of medical abortion between adolescents and adults. To eliminate the possible influence of previous pregnancies on the outcome of termination of pregnancy, we carried out a subgroup analysis among primigravid women. In addition we assessed the impact of a positive Chlamydia trachomatis test result at the time of 
abortion on the incidence of infections after abortiona situation of great clinical relevance to adolescents.

\section{METHODS}

From the national abortion register compiled by the National Institute for Health and Welfare we identified all women who had undergone induced abortion in Finland during 2000-6. The study population consisted of women who had had a medical abortion (mifepristone alone or in combination with misoprostol or other prostaglandins) at 20 weeks or less of gestation. We divided the women into two cohorts based on age at the time of abortion: adolescents ( $<18$ years) and adults ( $\geq 18$ years). To keep the observations independent, we included only the first abortion for women who had more than one during the study period. To assess the potential learning curve in the introduction of medical abortion, we analysed the results in part separately for the first years (2000-3) of its use compared with established use (2004-6). We linked the data with the care register for health institutions (later called the hospital register) and the national infectious diseases register, both compiled by the National Institute for Health and Welfare, and the cause of death register of Statistics Finland. We followed the women for 42 days after the induced abortion and linked all events recorded in the hospital register and cause of death register with the abortion register.

The Finnish national register on induced abortions and sterilisations has been maintained since 1977. In accordance with the current legislation, doctors performing induced abortions are obliged to report cases to the register within one month, using a specific data collection form. In Finland, data on induced abortions are collected from all hospitals and clinics that carry out induced abortions. The register contains data on women having termination of pregnancy. These data include information on pregnancy history, occupation, type of residence, municipality, and marital status. Data on current pregnancy include information on duration of gestation at the time of abortion, indication for abortion, and method of termination. ${ }^{5}$

We have previously described Finnish legislation on induced abortion. ${ }^{8}$ Briefly, current legislation permits termination of pregnancy of up to 20 weeks' gestation

\section{Classification of adverse events}

- Haemorrhage-all reported haemorrhage

- Infection-pelvic inflammatory disease, endometritis, cervicitis, wound infections, pyrexia of unknown origin, urinary tract infections, and septicaemia

- Any reported incomplete abortion

- Surgical evacuation

- Psychiatric morbidity—depression, intoxication, psychoses (ICD-10 codes F10-F48)

- Injury or other reason for surgical operation-all injuries, cervical laceration, uterine perforation, all surgical interventions during follow-up

- Thromboembolic disease-pulmonary embolism, deep vein thrombosis

- Death—death from any cause, pregnancy related death according to the World Health Organization definition
( 24 weeks in cases of a medical condition of the fetus) for social, medical, or ethical reasons. A national guideline on the care of women seeking abortion was published in 2001 and updated in 2007..$^{9}$ Based on this guideline all women should be screened for $C$ trachomatis and treated if it is present and screened for bacterial vaginosis at the first visit before the termination of pregnancy. Prophylactic antibiotics are not routinely used.

\section{Data collection}

All hospitals in Finland are required by law to provide the hospital register with information on inpatient treatment (all hospitals) and outpatient visits (public hospitals). This register contains information on diagnosis (international statistical classification of diseases and related health problems, ICD- $10^{10}$ ) and treatment (Nordic classification of surgical procedures ${ }^{11}$ ), as well as the dates of the treatment episodes. To analyse adverse events related to induced abortion we linked information on the study participants in the hospital register for all hospital inpatient episodes and outpatient visits within 42 days after termination of pregnancy with data in the abortion register. We selected diagnoses and codes for surgical procedures in the cohorts for those considered to be of clinical importance.

We divided the complications into eight categories (see box): haemorrhage, infection, incomplete abortion, surgical evacuation, psychiatric morbidity, injury or other reason for surgical operation, thromboembolic disease, and death. The classification was based on that reported in the joint study of the Royal College of General Practitioners and the Royal College of Obstetricians and Gynaecologists ${ }^{12}$ and modified for this and our previous study. ${ }^{6}$

The cause of death register contains data from death certificates and covers all deaths of Finnish citizens and permanent residents in Finland, classified according to ICD-10 codes. All the early deaths (within 42 days of termination of pregnancy) were classified as direct, indirect, or accidental. ${ }^{13}$

The National Department of Infectious Disease Epidemiology and Control at the National Institute for Health and Welfare collects information on cases of detected $C$ trachomatis infections. Since 1997 it has been mandatory for laboratories to report all positive cases to the national infectious diseases register based on the Communicable Diseases Act and Decree of $1987 .^{14}$ Since 2004, laboratory notifications have included personal identification numbers, enabling linkage of the data with that in other registries. Since 2004 genital $C$ trachomatis has been detected by DNA or RNA testing. ${ }^{14}$

\section{Statistical analysis}

To assess differences between the groups we used the Mann-Whitney test for age and the $\chi^{2}$ test for categorical variables. The $\chi^{2}$ test was also used to calculate the difference in the incidence of adverse events, except for rare ones (psychiatric morbidity, injury, thromboembolic disease, and death) when we used Fisher's 
Table 1 Characteristics of the two study cohorts. Values are numbers (percentages) unless stated otherwise

\begin{tabular}{|c|c|c|c|}
\hline Characteristics & $\begin{array}{l}\text { Adolescent cohort } \\
(<18 \text { years) }(n=3024)\end{array}$ & $\begin{array}{c}\text { Adult cohort } \\
(\geq 18 \text { years })(n=24 \text { 006) }\end{array}$ & $P$ value \\
\hline Mean (median) age (years), range & $16.1(16.0), 13-17$ & 27.6 (26.0), $18-50$ & $<0.001$ \\
\hline \multicolumn{4}{|l|}{ Previous pregnancies: } \\
\hline None & $2913(96.3)$ & $10474(43.6)$ & \multirow{2}{*}{$<0.001$} \\
\hline Yes & $111(3.7)$ & $13532(56.4)$ & \\
\hline \multicolumn{4}{|l|}{ Previous deliveries: } \\
\hline None & $2972(98.3)$ & $12059(50.2)$ & \multirow{2}{*}{$<0.001$} \\
\hline Yes & $52(1.7)$ & $11947(49.8)$ & \\
\hline \multicolumn{4}{|l|}{ Previous induced abortions: } \\
\hline None & 3004 (99.3) & $19432(80.9)$ & \multirow{2}{*}{$<0.001$} \\
\hline Yes & $20(0.7)$ & 4574 (19.1) & \\
\hline \multicolumn{4}{|l|}{ Marital status: } \\
\hline Married & $12(0.4)$ & $5634(23.5)$ & \multirow{4}{*}{$<0.001$} \\
\hline Cohabiting & $126(4.2)$ & $4546(18.9)$ & \\
\hline Single & $2882(95.3)$ & $13785(57.4)$ & \\
\hline Data missing & $4(0.1)$ & $41(0.2)$ & \\
\hline \multicolumn{4}{|l|}{ Type of residence: } \\
\hline Urban & $1979(65.4)$ & $17977(74.9)$ & \multirow{3}{*}{$<0.001$} \\
\hline Densely populated & $486(16.1)$ & $2986(12.4)$ & \\
\hline Rural & $559(18.5)$ & $3043(12.7)$ & \\
\hline \multicolumn{4}{|l|}{ Duration of gestation (weeks): } \\
\hline$<9$ & $2424(80.2)$ & 20143 (83.9) & \multirow{5}{*}{$<0.001$} \\
\hline $9-12$ & $139(4.6)$ & $660(2.7)$ & \\
\hline $13-16$ & $283(9.4)$ & $1741(7.3)$ & \\
\hline $17-20$ & $171(5.7)$ & $1151(4.8)$ & \\
\hline Data missing & $7(0.2)$ & $311(1.3)$ & \\
\hline Chlamydia trachomatis positive test result* & $99 / 1749(5.7)$ & $496 / 13547(3.7)$ & $<0.001$ \\
\hline
\end{tabular}

*Data available for 2004-6. more often single and living in a non-urban setting. In both groups, most of the medical abortions (over $80 \%$ ) were performed before nine weeks of gestation, but the mean duration of gestation was more advanced among adolescents. The incidence of $C$ trachomatis infections, diagnosed four weeks before to six weeks after abortion, was higher in the adolescent cohort, as calculated for 2004-6.

Table 2 describes the incidence of adverse events among the two cohorts, as well as among the primigravid women. The adult cohort had a significantly higher incidence of haemorrhage (3690 (15.4\%) v386 (12.8\%), $\mathrm{P}<0.001)$, incomplete abortion $(2450(10.2 \%)$ v 212 $(7.0 \%), \mathrm{P}<0.001)$, and surgical evacuation of retained products of conception (3121 (13.0\% v 333 (11.0\%), $\mathrm{P}=0.002$ ). Odds ratios were calculated for main adverse events (haemorrhage, infection, incomplete abortion, and surgical evacuation), after adjustment for parity, previous abortions, marital status, type of residence, duration of gestation, and year of abortion. In the adolescent cohort the adjusted odds ratios were significantly lower for haemorrhage, incomplete abortion, and surgical evacuation than in the adult cohort. In addition, the adult cohort had more participants with adverse events (5535 (23.1\%) v 575 (19.0\%), $\mathrm{P}<0.001)$.

In the subgroup analysis carried out among the primigravid women, the proportion of women with haemorrhage $(1505(14.4 \%)$ v $374(12.8 \%), \mathrm{P}=0.035)$, incomplete abortions (887 (8.5\%) v 201 (6.9\%), $\mathrm{P}=0.006)$ and a higher overall number of adverse events $(2224(21.1 \%)$ v $552(18.9 \%), \mathrm{P}=0.031)$ was significantly higher in the adult cohort. After adjustment for marital status, type of residence, duration of gestation, and year of abortion, the risks for incomplete abortion and surgical evacuation were lower in the primigravid adolescents than in the primigravid adults (table 2).

The incidence of a psychiatric diagnosis was higher among the adolescents in both the cohort and the primigravid cohort, even though the overall numbers were low. Two deaths were reported during the follow-up period. Both of these occurred in adults and were unrelated to the pregnancy (intracranial trauma and melanoma).

The figure shows the results of logistic regression among the primigravid women for risk of main adverse events (haemorrhage, infection, incomplete abortion, and surgical evacuation). An increased risk of haemorrhage was associated with living in a densely populated area. The risk of bleeding after medical abortion was higher during 2004-6 than during 2000-3. Gestations of 9-12 or 13-16 weeks were associated with a lower risk of haemorrhage than gestations of less than nine weeks. The risk of haemorrhage was also significantly lower in the adolescent cohort.

Advanced duration of gestation (9-12, 13-16, and 17-20 weeks) was associated with an increased risk of infections after abortion (figure). Additionally, being married or cohabiting compared with being single was associated with an increased risk of infection. 
Table 2| Incidence of adverse events in study cohorts for all women (3024 adolescents and 24006 adults) and for primigravid women (2913 adolescents and 10474 adults)

\begin{tabular}{|c|c|c|c|c|c|c|}
\hline Adverse events & $\begin{array}{c}\text { Adolescent } \\
\text { cohort } \\
\text { ( }<18 \text { years) }\end{array}$ & $\%(95 \% \mathrm{Cl})$ & $\begin{array}{l}\text { Adult cohort } \\
\text { ( } \geq 18 \text { years) }\end{array}$ & $\%(95 \% \mathrm{Cl})$ & $P$ value & $\begin{array}{c}\text { Adjusted odds ratio } \\
(95 \% \mathrm{Cl})^{\star}\end{array}$ \\
\hline \multicolumn{7}{|l|}{ All women } \\
\hline Haemorrhage & 386 & $12.8(11.6$ to 14.0$)$ & 3690 & 15.4 (15.0 to 16.0$)$ & $<0.001 \dagger$ & $0.87(0.77$ to 0.99$) \dagger$ \\
\hline Infection & 60 & 2.0 (1.5 to 2.6$)$ & 489 & 2.0 (1.9 to 2.2 ) & 0.742 & $0.97(0.73$ to 1.30$)$ \\
\hline Incomplete abortion & 212 & $7.0(6.1$ to 8.0$)$ & 2450 & $10.2(9.8$ to 10.6$)$ & $<0.001 \dagger$ & $0.69(0.59$ to 0.82$) \dagger$ \\
\hline Surgical evacuation & 333 & $11.0(9.9$ to 12.1$)$ & 3121 & 13.0 (12.6 to 13.4$)$ & $0.002 \dagger$ & $0.78(0.67$ to 0.90$) \dagger$ \\
\hline Psychiatric morbidity & 3 & $0.10(0.02$ to 0.29$)$ & 2 & NA & $0.012 \dagger$ & - \\
\hline Injury & 4 & $0.13(0.04$ to 0.34$)$ & 35 & 0.15 (0.10 to 0.19$)$ & 1.000 & - \\
\hline Thromboembolic disease & 2 & $0.07(0.01$ to 0.24$)$ & 26 & 0.11 (0.07 to 0.15$)$ & 0.764 & - \\
\hline Death & 0 & NA & 2 & NA & 0.392 & - \\
\hline \multicolumn{7}{|c|}{ No of adverse events per woman: } \\
\hline 0 & 2449 & $81.0(79.6$ to 82.4$)$ & 18471 & 76.9 (76.4 to 77.5$)$ & \multirow{5}{*}{$<0.001 \dagger$} & - \\
\hline 1 & 488 & 16.1 (14.8 to 17.4$)$ & 4456 & 18.6 (18.1 to 19.1$)$ & & - \\
\hline 2 & 82 & $2.7(2.2$ to 3.4$)$ & 994 & $4.1(3.9$ to 4.4$)$ & & - \\
\hline 3 & 5 & $0.17(0.05$ to 0.39$)$ & 83 & $0.35(0.27$ to 0.42$)$ & & - \\
\hline 4 & 0 & NA & 2 & NA & & - \\
\hline \multicolumn{7}{|l|}{ Primigravid women } \\
\hline Haemorrhage & 374 & $12.8(11.6$ to 14.1$)$ & 1505 & $14.4(13.7$ to 15.0$)$ & $0.035 \dagger$ & $0.88(0.78$ to 1.00$)$ \\
\hline Infection & 57 & $2.0(1.5$ to 2.5$)$ & 227 & 2.2 (1.9 to 2.5$)$ & 0.486 & $1.01(0.75$ to 1.37$)$ \\
\hline Incomplete abortion & 201 & $6.9(6.0$ to 7.9$)$ & 887 & 8.5 (7.9 to 9.0$)$ & $0.006 \dagger$ & $0.68(0.56$ to 0.81$) \dagger$ \\
\hline Surgical evacuation & 311 & 10.7 (9.6 to 11.8$)$ & 1136 & $10.8(10.3$ to 11.4$)$ & 0.794 & $0.75(0.64$ to 0.88$) \dagger$ \\
\hline Psychiatric morbidity & 3 & $0.10(0.02$ to 0.30$)$ & 1 & NA & $0.034 \dagger$ & - \\
\hline Injury & 4 & $0.14(0.04$ to 0.35$)$ & 10 & $0.10(0.04$ to 0.16$)$ & 0.521 & - \\
\hline Thromboembolic disease & 2 & $0.07(0.01$ to 0.25$)$ & 10 & $0.10(0.04$ to 0.16$)$ & 1.00 & - \\
\hline Death & 0 & NA & 1 & NA & 0.391 & - \\
\hline \multicolumn{7}{|c|}{ No of adverse events per woman: } \\
\hline 0 & 2361 & 81.1 (79.6 to 82.5$)$ & 8250 & 78.8 (78.0 to 79.5$)$ & \multirow{5}{*}{$0.031 \dagger$} & - \\
\hline 1 & 468 & $16.1(14.7$ to 17.4$)$ & 1838 & 17.5 (16.8 to 18.3$)$ & & - \\
\hline 2 & 79 & 2.7 (2.2 to 3.4$)$ & 356 & 3.4 (3.1 to 3.8$)$ & & - \\
\hline 3 & 5 & $0.17(0.06$ to 0.40$)$ & 30 & 0.29 (0.18 to 0.39$)$ & & - \\
\hline 4 & 0 & NA & 0 & NA & & - \\
\hline
\end{tabular}

$N A=$ not applicable owing to small number of women.

*Adult cohort as reference for all women adjusted for parity, previous abortions, marital status, type of residence, duration of gestation, and year of abortion; adult cohort as reference for primigravid women adjusted for marital status, type of residence, duration of gestation, and year of abortion.

†Statistically significant.

Also, the risk was higher in the later period (2004-6) than in 2000-3. The risk of infection was similar between the two cohorts.

Advanced duration of gestation was strongly related to the risk of incomplete abortion and surgical evacuation. The risk of incomplete abortion was lower in adolescents (odds ratio $0.69,95 \%$ confidence interval 0.58 to 0.82 ) than in adults. The risk of surgical evacuation was increased in women living in rural areas and in those who were married or cohabiting. When abortion was carried out in the later period (2004-6) the risk of surgical evacuation was diminished (figure).

The risk of infections after abortion as a result of concurrent chlamydia infection was assessed among women who underwent abortion during 2004-6. In logistic regression analysis of the whole cohort, the risk of infection after abortion was not associated with concurrent chlamydia infection (1.02, 0.58 to 1.78$)$. Moreover, no significant difference in the rate of infections after abortion emerged between adolescents and those with a positive test result for $C$ trachomatis (data not shown).

\section{DISCUSSION}

In the present study the rate of adverse events and complications after medical abortion in adolescents was similar to or lower than that in adults. Various characteristics of the two cohorts differed significantly (table 1), but the risk of adverse events was calculated after adjustment for these factors. This study covered almost all abortions carried out in Finland in all regions and hospitals during a seven year period and thus shows reliable national trends. Earlier studies assessing the completeness of the Finnish abortion register found that $99 \%$ of abortions were reported to the register and at least $95 \%$ of information matched the medical records. ${ }^{1617}$

One limitation of the study is that the registry based data lack detailed information as the diagnoses were made on clinical grounds, and the severity of adverse 


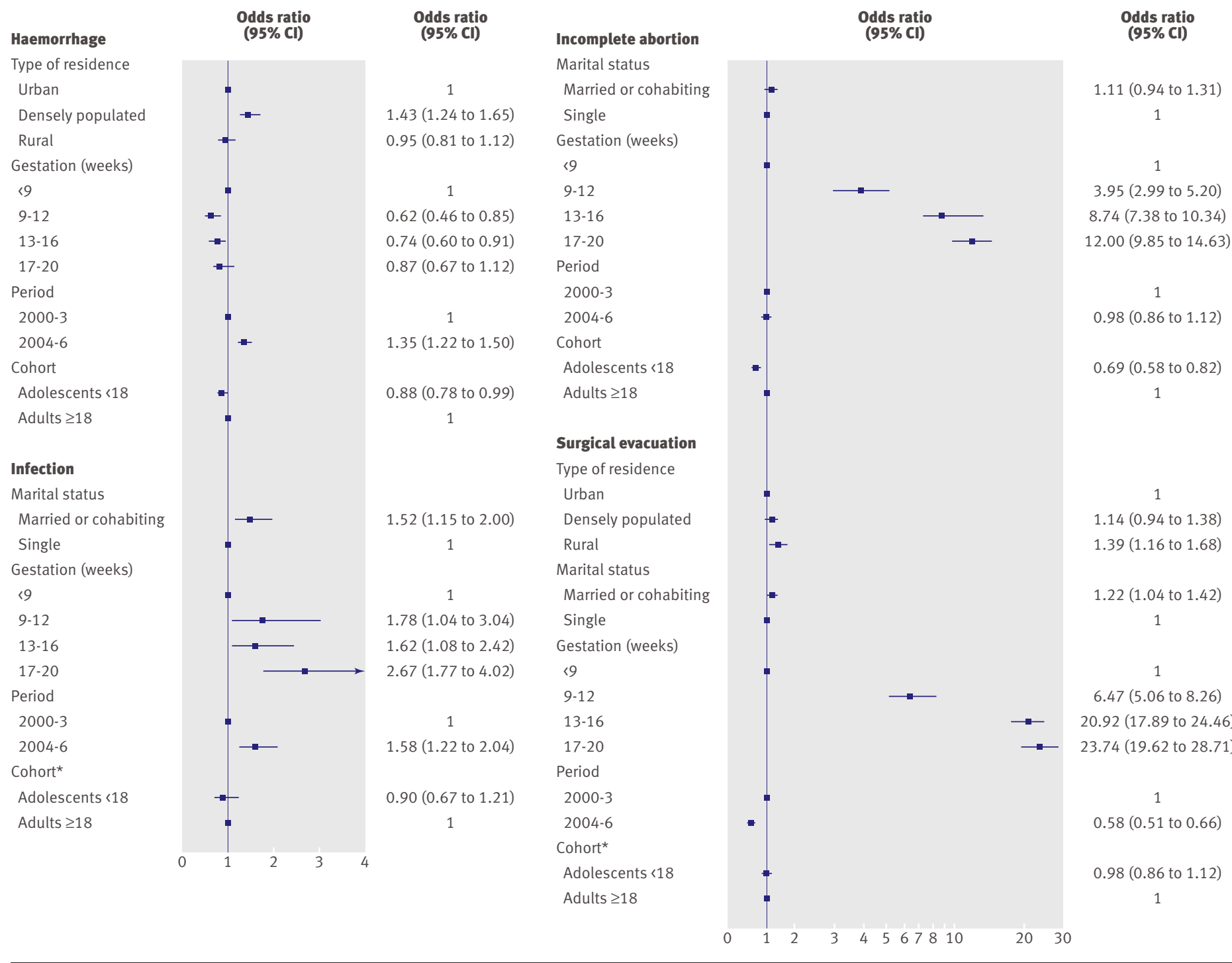

Logistic regression analysis of risk factors for main adverse events (haemorrhage, infection, incomplete abortion, and surgical evacuation) among primigravid women in entire cohort. Results of multivariate analysis are shown unless stated otherwise. Variables showing significance in univariate analysis are included. *Derived from univariate analysis

events may vary substantially. Another drawback is that no conclusions can be made on the effects of abortion beyond the 42 days of follow-up. A further limitation is that data on $C$ trachomatis could only be linked with registry data from 2004, when identification numbers were first archived.

More women sought help for bleeding after abortion when gestation was less than nine weeks. This finding parallels that reported in our previous study. ${ }^{6}$ This might be explained partly by the fact that medical abortions at nine weeks or more of gestation are carried out by hospitals, and not on an outpatient basis. ${ }^{9}$ Moreover, an increasing number of these early abortions are carried out at home using self administered misoprostol.

The risk of surgical evacuation of retained products after medical abortion decreased during 2004-6 compared with 2000-3, whereas the number of incomplete abortions remained the same. These findings probably reflect a learning curve in providing medical abortion. However, the lower number of surgical evacuations occurred at the expense of an increased rate of consultations as a result of uterine bleeding. We took into account the possible bias caused by the differences between the study periods (2000-3 and 2004-6) by adjusting the odds ratios of adverse events by study period.

The rate of infections after abortion was higher $(2.0 \%)$ than that reported in an earlier review in which medical abortion was assessed $(0.9 \%) .{ }^{18}$ The higher figure may in part be a result of the register based nature of the present study - that is, the diagnostic criteria lacked uniformity. In recent reviews, however, the incidence of infections after medical abortion in the second trimester has been estimated to be about 3\%. ${ }^{1920}$ Thus in the present study, concerning pregnancies of up to 20 weeks' duration, the incidence of infections was comparable with that reported in the recent 


\section{WHAT IS ALREADY KNOWN ON THIS TOPIC}

Teenage pregnancies are mostly unplanned and often result in induced abortion

Medical abortion is increasingly used, albeit its safety has not been properly assessed among adolescents

\section{WHAT THIS STUDY ADDS}

The risk of adverse events (haemorrhage, incomplete abortion, infection) after medical abortion is similar or even lower in adolescent ( $(18$ years) compared with adult women

reviews. The risk of infection was increased when the abortion was carried out in the later period (2004-6). The explanation for this is unclear. The incidence of $C$ trachomatis infections in the Finnish population did not change at the same time. ${ }^{14}$

$C$ trachomatis is a notable cause of pelvic inflammatory disease. Screening for and treatment of $C$ trachomatis can prevent the development of the disease after abortion. ${ }^{21}$ To prevent infection after termination of pregnancy both prophylactic antibiotic therapy for all and screen and treat strategies are in use. In a recent study in the United States, routine provision of doximycin at the time of medical abortion was associated with a significant reduction in the rate of serious infections. ${ }^{22}$

We found no correlation between $C$ trachomatis diagnosed at the time of abortion and subsequent infections. In Finland, systematic screening for $C$ trachomatis after termination of pregnancy is enforced by national guidelines. ${ }^{9}$ In 2004- 6 the national incidence of $C$ trachomatis among girls and young women aged $10-19$ was $1.7 \%$ in Finland, ${ }^{14}$ whereas a higher rate of $5.7 \%$ was detected in the present adolescent cohort. The results of this study do not rule out the possible association with infections after abortions in the cases of untreated $C$ trachomatis infections, or with delayed antibiotic treatment. The present study suggests that by timely screening it is possible to treat the infection before the clinical manifestation.

In the present study psychiatric morbidity was significantly more common among adolescents than among adults, although the number of cases was small. Register based studies are not ideal for studying psychiatric disorders, as only some women seek professional help for mental disorders and only some women with mental disorders are treated in specialised healthcare. In a recent register based Danish study, the risk of a psychiatric disorder in women with no such previously detected disorders was not increased after induced abortion in the first trimester. ${ }^{23}$ The risk of psychiatric contact was not, however, significantly affected by age. In a US survey, adolescents were not at increased risk for depression or lower self esteem after abortion than the controls during follow-up. ${ }^{24}$ The present studies only assessed psychiatric diagnoses during the short follow-up but not possible psychiatric morbidity before abortion. Thus the association of mental disorders and termination of pregnancy among adolescents remains unresolved.
Experience of pain or satisfaction with care could not be studied in the present setting, as these outcomes are not registered in the Finnish abortion register. In a randomised study, women with higher gestational age and first pregnancy seemed to be less satisfied with medical abortion as a result of more pain during the termination. ${ }^{25}$ The effective treatment of pain must be taken into account when adolescents, predominantly nulliparous women, undergo induced abortion.

\section{Conclusion}

The present population based national study provides evidence that medical abortion is not associated with additional risks of adverse events among adolescents in the short term compared with adult women. The data were derived from one country with a homogeneous population but can be generalised to populations with high quality healthcare and easy access to specialist treatment.

The preliminary results of this study were presented at the International Federation of Obstetrics and Gynecology (FIGO) meeting in Cape Town, South Africa October 2009, and in the International Federation of

Professional Abortion and Contraception Associates (FIAPAC) meeting in Seville Spain, October 2010 (MN). We thank Aini Bloigu (National institute for Health and Welfare, Oulu, Finland) for her professional help with the statistics.

Contributors: All authors participated in the design of the study. MN carried out the data analysis, wrote the first draft of the manuscript, and is a guarantor of the study. All authors contributed to the subsequent writing of the paper and gave substantial input into the study. $\mathrm{OH}$ obtained funding for the study. MG is in charge of the Finnish reproductive registries (including the abortion register).

Funding: This study was funded by Finnish Cultural Foundation (MN), Helsinki University Central Hospital Research Funds $(\mathrm{OH}, \mathrm{MN})$, and University Hospital of Oulu Research Funds (MN).

Competing interests: All authors have completed the Unified Competing Interest form at www.icmje.org/coi_disclosure.pdf (available on request from the corresponding author) and declare: $\mathrm{OH}$ has lectured at an educational event organised by Nordic Drugs and has been principal investigator in clinical studies sponsored by the Concept Foundation; no financial relationships with any organisations that might have an interes in the submitted work in the previous three years, no other relationships or activities that could appear to have influenced the submitted work. Ethical approval: This study was approved by the ethics committee of the Northern Ostrobothnia Hospital District in October 2005 (No 46/2005). The Ministry of Social Affairs and Health, and Statistics Finland gave permission for the use of confidential personal level data from the registries. The data protection ombudsman was notified about the data linkage before the analyses, as required by national data protection legislation.

Data sharing: No additional data available.

1 Jones RK, Finer LB, Singh S. Characteristics of US abortion patients, 2008. Guttmacher Institute, 2010.

2 Sihvo S, Bajos N, Ducot B, Kaminski M. Women's life cycle and abortion decision in unintended pregnancies. J Epidemiol Community Health 2003;57:601-5.

3 Department of Health. Abortion statistics, England and Wales: 2009. 2010. www.dh.gov.uk/en/Publicationsandstatistics/Publications/ PublicationsStatistics/DH_116039.

4 Socialstyrelsen. Statistics. 2010. www.socialstyrelsen.se/statistics.

5 National Institute for Health and Welfare. Induced abortions. 2010. www.stakes.fi/EN/tilastot/statisticsbytopic/reproduction/ abortions.htm.

6 Niinimäki M, Pouta A, Bloigu A, Gissler M, Hemminki E, Suhonen S, et al. Immediate complications after medical compared with surgical termination of pregnancy. Obstet Gynecol 2010;115:660-1.

7 Phelps RH, Schaff EA, Fielding SL. Mifepristone abortion in minors. Contraception 2001;64:339-43.

8 Niinimäki M, Pouta A, Bloigu A, Gissler M, Hemminki E, Suhonen S, et al. Frequency and risk factors for repeat abortions after surgical 
compared with medical termination of pregnancy. Obstet Gynecol 2009;113:845-52.

9 Finnish Gynecological Association's Task Force. Abortion. Duodecim 2001;117:2084-94.

10 World Health Organization. International Classification of Diseases (ICD). 2010. www.who.int/classifications/icd/en/.

11 Nordic Centre for Classifications in Health Care. NCSP-the NOMESCO classification of surgical procedures. 2010. www. helsedirektoratet.no/nordclass_english/ncsp/

ncsp the nomesco_classification_of_surgical_proce dures_334824.

12 RCGP/RCOG. Induced abortion operations and their early sequelae. Joint study of the Royal College of General Practitioners and the Roya College of Obstetricians and Gynaecologists. J R Coll Gen Pract 1985;35:175-80.

13 Deneux-Tharaux C, Berg C, Bouvier-Colle MH, Gissler M, Harper M, Nannini A, et al. Underreporting of pregnancy-related mortality in the United States and Europe. Obstet Gynecol 2005;106:684-92.

14 National Institute for Health and Welfare. National Department of Infectious Disease Epidemiology. The statistical database of the infectious diseases register. 2010. www3.ktl.fi/.

15 Gardner MJ, Altman DG. Statistics with confidence. Confidence intervals and statistical guidelines. BMJ Publishing, 1989

16 Gissler M, Ulander VM, Hemminki E, Rasimus A. Declining induced abortion rate in Finland: data quality of the Finnish abortion register. Int J Epidemiol 1996;25:376-80.
17 Heikinheimo O, Gissler M, Suhonen S. Age, parity, history of abortion and contraceptive choices affect the risk of repeat abortion. Contraception 2008;78:149-54.

18 Shannon C, Brothers LP, Philip NM, Winikoff B. Infection after medical abortion: a review of the literature. Contraception 2004;70:183-90.

19 Gemzell-Danielsson K, Lalitkumar S. Second trimester medical abortion with mifepristone-misoprostol and misoprostol alone: a review of methods and management. Reprod Health Matters 2008;16(suppl 21):162-72.

20 Lohr PA, Hayes JL, Gemzell-Danielsson K. Surgical versus medical methods for second trimester induced abortion. Cochrane Database Syst Rev 2008;1:CD006714.

21 Soper DE. Pelvic inflammatory disease. Obstet Gynecol 2010;116:419-28.

22 Fjerstad M, Trussell J, Sivin I, Lichtenberg ES, Cullins V. Rates of serious infection after changes in regimens for medical abortion. $N$ Engl J Med 2009;361:145-51.

23 Munk-Olsen T, Laursen T, Pedersen CB, Lidegaard O, Mortensen PB. Induced first-trimester abortion and risk of mental disorder. N Engl Med 2011;364:332-9.

24 Warren JT, Harvey SM, Henderson JT. Do depression and low selfesteem follow abortion among adolescents? Evidence from a national study. Perspect Sex Reprod Health 2010;42:230-5.

25 Teal SB, Dempsey-Fanning A, Weshoff C. Predictors of acceptability of medication abortion. Contraception 2007;75:224-9.

Accepted: 20 February 2011 